\title{
Non-COVID-19 In-Hospital Admission in a Large Academic Center in Belgium During the First Two Waves of the COVID-19 Pandemic
}

\author{
Jean Cyr Yombi' \\ Halil Yildiz (D) \\ Claire Beguin ${ }^{2}$ \\ Laurence Habimana ${ }^{2}$ \\ 'Department of Internal Medicine and \\ Infectious Diseases, Cliniques \\ Universitaires Saint-Luc, Brussels, 1200 , \\ Belgium; ${ }^{2}$ Department of Medical \\ Informatics and Statistics, Cliniques \\ Universitaires Saint-Luc, UCLouvain, \\ Brussels, 1200, Belgium
}

Background: Preliminary reports described a reduction in non-COVID admissions during the first wave of the pandemic including some of critical diseases such as cancer, myocardial and cerebral infarction.

Objective: The aim of our study was to evaluate the impact of the COVID-19 pandemic on non-COVID in-hospital admissions in a large academic center in Belgium.

Materials and Methods: We performed a retrospective study of non-COVID-19 inhospital admissions during the first two waves of the COVID-19 pandemic. The average number of admissions per week in 2020 has been compared to that of the same period in 2019 and 2018. Comparisons were made first for all admissions, then by disease groups, using the classification of APRDRG, and then by diagnoses using ICD-10-CM classification. Results: Overall in-hospital admissions were reduced by around 39\% and $29 \%$ during the first and the second waves of the COVID-19 pandemic respectively compared to 2018 and 2019. No significant difference was found between the average number of admissions in the early-COVID and the pre-COVID baseline period during the two waves. The average number of admissions was significantly reduced in the peak-COVID period compared to the baseline (first wave: 332 versus 763 admissions/week, p<0.01, $-57 \%$; second wave: 496 versus 788 admissions/week, $\mathrm{p}<0.01,-37 \%$ ), as well as in the late-COVID period compared to the baseline (first wave: 412 versus 763 admissions/week, $\mathrm{p}<0.01,-46 \%$; second wave: 470 versus 788 admissions/week, $\mathrm{p}<0.01,-40 \%$ ). Cancer, myocardial and cerebral infarction admissions were not statistically reduced during the the two waves of COVID pandemic compared to the pre-COVID period.

Conclusion: Our study shows that non-COVID in-hospital admissions rates were substantially reduced during the first two waves of COVID-19 pandemic. In our study, cancer, myocardial and cerebral infarction admissions were not statistically reduced, which was not in accordance to what was described in the literature.

Keywords: COVID-19 pandemic, non-COVID-19 admissions, SARS-COV-2
Correspondence: Jean Cyr Yombi Department of Internal Medicine and Infectious Diseases, Cliniques

Universitaires Saint-Luc, 10 Avenue Hippocrate, Brussels, 1200, Belgium Tel +3227641902

Fax +3227641046

Email Jean.yombi@uclouvain.be

\section{Introduction}

The Coronavirus disease 2019 (COVID-19) pandemic due to severe acute respiratory syndrome virus 2 (SARS-CoV-2) resulted in significant pressure on the health care systems of countries around the world. ${ }^{1-3}$ Hospitals were rapidly overwhelmed by the number of COVID-19 patients requiring hospital care, and by the need to control the spread of the disease. To try to respond to this pandemic, the majority of hospitals adopted special measures, including work reorganization, creation of 
dedicated COVID wards and resource reallocation. ${ }^{1-3}$ At the countries level, many governments enforced restrictive measures to control the spread of the disease, including among others lockdowns, the closing of schools and nonessential commercial activities, non-essential travel restrictions and social distancing. In Belgium on March 13, 2020, a complete lockdown was declared. This lockdown led to a modification of routine hospital practices with the consequences of postponing or canceling admissions of non-COVID-19 patients for whom non-urgent care was not necessary. The consequences of this strategy on the clinical outcomes and quality of life of non-COVID-19 patients are not completely evaluated or understood. Many reports suggest that even patients with life-threatening conditions may have avoided in-hospital admission, because of the fear of SARS-CoV-2 potential exposure or other non-understood reasons. ${ }^{4-8}$ Preliminary reports described in some countries a reduction on non-COVID in-hospital admissions during the first wave of the pandemic including some of critical diseases such as cancer, myocardial and cerebral infarctions. ${ }^{9-15}$

Belgium is among countries severely hit by the COVID-19 pandemic. During the first wave, Belgium was ahead of the United Kingdom, Spain, Italy and Sweden in terms of deaths related to COVID-19 per 100,000 inhabitants. However, data on the impact of restrictive measures on non-COVID patients in-hospital admissions in our country are not published.

The aim of our study was to evaluate the impact of the COVID-19 pandemic on non-COVID in-hospital admissions (all admissions and admissions per group of diseases) during the first and second waves in a large academic center in Belgium.

\section{Materials and Methods}

\section{Context and Setting}

In Belgium on March 13, 2020, a complete lockdown was declared. All hospitals have been asked to activate their emergency plan to be able to welcome all COVID-19 patients. This involved increasing their capacity in the intensive care unit (ICU), and postponing all non-urgent consultations, examinations and elective interventions. During the second wave, an adaptive plan was developed to manage the occupation of beds from October and depending on the evolution of the pandemic. However, on October 30, 2020, due to a high increase in the number of contamination, the government declared a second lockdown. All hospitals were again asked to activate their emergency plan (non-urgent consultations were not cancelled this time).

The Cliniques Universitaires Saint-Luc (CUSL) is one of the largest teaching hospitals with a capacity of 973 beds, located in Brussels, Belgium. There are around 39,000 in-hospital admissions each year, or 750 admissions per week. In our hospital, we have made the following decisions regarding admissions: all the patients admitted to hospital were tested by PCR on nasopharyngeal swab to rule out SARS-CoV-2 infection. Wearing a mask was compulsory for all nursing staff during and outside care. We created two separate channels for radiological investigation, one for COVID patients and another for non-COVID patients. An operating theater dedicated specifically to COVID patients was created. During the first wave, vital surgical and oncological admissions were maintained from March to May 2020, however all non-urgent surgeries and surgeries performed in one-day clinic were canceled. Every surgeon was also asked on a case-to-case basis to select himself what he considered as urgent surgery every week and to postpone what he considered as non-urgent. The final decision was taken by the institutional board of COVID management. Surgical activities were then gradually resumed, first at $55 \%$ in the first half of May 2020 then $75 \%$ in the second half of May 2020 and for a recovery at $100 \%$ in June 2020. Concerning non-surgical admissions, activities in gastroenterology and neurology other than stroke have been reduced, as have alcohol withdrawal activities. During the second wave, admissions for surgery were managed as follows: from October 26, 2020 we halved access to the operating room with maintenance of all vital and oncological emergencies, and from the beginning of November to a third of the operative activity, and to return to a full activity in December 2020. For non-surgical admissions we stopped only the alcohol withdrawal activities and we resumed full activity at the end of December.

\section{Methods}

We analyzed retrospectively non-COVID-19 in-hospital admissions, using data from minimum clinical summary from patient charts. Admissions for COVID-19 reasons were excluded, based on diagnostic codes (ICD-10-CM classification: B9729). The average number of admissions per week in 2020 has been compared to that of the same period in 2019 and 2018. Admissions in 2020 have been separated between the first wave of the COVID-19 pandemic: from 
March 1, 2020 to May 9, 2020 and the second wave: from September 6, 2020 to December 19, 2020. During each wave, we looked at three different time periods, depending on the incidence of admissions for COVID-19: an early period (early-COVID period: March 1, 2020 to March 21, 2020; September 6, 2020 to October 10, 2020), a peak period (peak-COVID: from March 22, 2020 to April 11, 2020; October 11, 2020 to November 14, 2020) and a late period (late-COVID: from April 12, 2020 to May 30, 2020; November 15, 2020 to December 19, 2020), these three periods being compared to a baseline period without COVID (pre-COVID period). The pre-COVID period consists of the calendar weeks of 2018 and 2019 mirroring those of 2020 which were used to define the periods of interest for the COVID-19 epidemic. The weeks of 2018 and 2019 are pooled by period of interest to form an identical group, the number of admissions entered for the baseline is therefore an average of 2018 and 2019. Comparisons were made first for all admissions; then by disease groups, using the classification of APRDRG (All patient Refined Diagnosis Related Group) in version 34 (the thirty most frequent APRDRGs during the pre-COVID period); then by diagnosis categories (the thirty most frequent admission groups of diagnoses during the pre-COVID period), using the 3 first-digit codes according to the International Classification of Diseases (ICD-10-CM).

\section{Inclusion and Exclusion Criteria}

All in-hospital admissions of the same selected periods of interest in 2018, 2019 and 2020 were included, except COVID-19 admissions, based on diagnosis code (ICD-10CM classification: B9729).

\section{Ethical Issues}

The institutional ethical board approved the study (CEHF 2020/06AVR/201, Comité d'Ethique Hospitalo-Facultaire, Cliniques Universitaires Saint-Luc). Since it was a retrospective study, an informed consent was not necessary. The study was performed in accordance with guidelines outlined in the Declaration of Helsinki, and confidentiality of patients was guaranteed.

\section{Outcomes}

The first objective was to compare the number of nonCOVID in-hospital admissions during the two waves of the COVID-19 pandemic with admissions during a similar period in 2018 and 2019.
The second objective was to analyse the impact of COVID pandemic on in-hospital admissions for diagnosis considered as the most frequent during the pre-COVID period (2018 and 2019).

\section{Statistical Analysis}

In-hospital admissions were considered continuous and results were expressed as means, without decimals. We used parametric tests thanks to the large number of admissions, even in the subgroups. We performed analysis of variance, with the SIDAK correction for multiple comparisons, assuming a level of statistical significance of $<0.01$. Post-hoc comparisons were made between the average number of admissions per week during the early-COVID and the pre-COVID period (p-value ${ }^{\mathrm{a}}$ in tables), then between the peak-COVID and the pre-COVID period ( $\mathrm{p}-$ value $^{\mathrm{b}}$ in tables), and between the late-COVID period and the peak -COVID period ( $p$-value ${ }^{c}$ in tables). Analyses were implemented using SAS for Windows version 9.4. The charts were produced using Microsoft Excel 2016.

\section{Results}

\section{Primary Outcome}

During the first wave of the COVID-19 pandemic, between March 1, 2020 and May 9, 2020, there were 4608 non-COVID admissions in Cliniques Universitaires Saint Luc, compared to 7649 admissions in 2018 and 7614 in 2019; this corresponds to a reduction of $39 \%$ of admissions per week during the first wave compared to 2018 and 2019 (461 admissions per week, and 765 and 761, respectively in 2018 and 2019: $\mathrm{p}<0.01$ ).

During the second wave, between September 6 and December 19, 2020, there were 8378 non-COVID admissions, compared to 11,768 and 11,876 admissions in 2018 and 2019; this corresponds to a reduction of $29 \%$ of admissions per week during the second wave compared to 2018 and 2019 (558, 784 and 792 admissions per week during the second wave, in 2018 and in 2019 respectively: $\mathrm{p}<0.01)$.

According to the defined and so-called periods of the COVID-19 (early, peak and late) (Table 1).

The average number of admissions per week in the early-COVID period was not statistically different in the two waves of COVID from the pre-COVID baseline period (First wave: 654 admissions/week versus 763, $\mathrm{p}=0.15$, reduction of 14\%; Second wave: 709 admissions/week versus $788, \mathrm{p}=0.02$, reduction of $10 \%$ ). 
Table I Admissions per Week by Period of Interest During First and Second Waves of COVID-I9

\begin{tabular}{|c|c|c|c|c|c|c|c|c|}
\hline \multirow[t]{2}{*}{ COVID Waves } & \multicolumn{8}{|c|}{ Mean Hospitalizations per Week } \\
\hline & Baseline & Early COVID-I9 & $P$ value $^{\mathrm{a}}$ & Peak COVID-I9 & $P$ value ${ }^{b}$ & Late COVID-19 & $P$ value ${ }^{c}$ & $P$ value $^{\text {d }}$ \\
\hline First Wave & 763 & 654 & 0.15 & 332 & $<0.01$ & 412 & 0.68 & $<0.01$ \\
\hline Second wave & 788 & 709 & 0.03 & 496 & $<0.01$ & 470 & 0.97 & $<0.01$ \\
\hline
\end{tabular}

Notes: ${ }^{a}$ Comparison of early COVID-19 admissions to baseline admissions. ${ }^{b}$ Comparison of peak COVID-19 admissions to baseline admissions. ${ }^{\mathrm{C}}$ Comparison of late COVID-19 admissions to peak COVID-19 admissions. ${ }^{\mathrm{d}}$ Comparison of late COVID-19 admissions among all the periods.

The average number of admissions per week in the peak-COVID period was significantly lower from the preCOVID baseline period (First wave: 332 admissions/week versus $763, \mathrm{p}<0.01$, reduction of $56 \%$; Second wave: 496 admissions/week versus $788, \mathrm{p}<0.01$, reduction of $37 \%$ ).

The average number of admissions per week in the late-COVID period was significantly lower from the preCOVID baseline period (First wave: 412 admissions/week versus $763, \mathrm{p}<0.01$, reduction of $46 \%$; Second wave: 470 admissions/week versus $788, \mathrm{p}<0.01$, reduction of $40 \%$ ).

\section{Secondary Outcome}

\section{Analyses by APRDRG Classification}

Considering the disease groups classification (APRDRG), we found no significant decrease in the average number of admissions per week between the early and the preCOVID period, during the first two waves (Table 2, Figure 1A and B [Supplementary File]).

During the peak-COVID period, the following APRDRGs decreased significantly compared to the baseline pre-COVID period: Other disorders of the nervous system (First wave: 1 vs 7 admissions per week, $\mathrm{p}<0.01$, reduction of $85 \%$ ); Eye procedures except orbit (First wave: 2 vs 8 admissions per week, $\mathrm{p}=0.01$, reduction of $76 \%$; Second wave: 3 vs 7 admissions per week, $\mathrm{p}<0.01$, reduction of $55 \%$ ); Other ear, nose, mouth and throat procedures (First wave: 2 vs 9 admissions per week, $\mathrm{p}<0.01$, reduction of $83 \%$; Second wave: 4 vs 10 admissions per week, $\mathrm{p}<0.01$, reduction of $59 \%$ ); Other ear, nose, mouth, throat and cranial or facial diagnoses (First wave: 3 vs 12 admissions per week, $\mathrm{p}<0.01$, reduction of $79 \%$; Second wave: 7 vs 17 admissions per week, $\mathrm{p}<0.01$, reduction of 58\%); Cardiac valve procedures without acute myocardial infarction or complex procedures and diagnoses (Second wave: 2 vs 6 admissions per week, $\mathrm{p}<0.01$, reduction of $69 \%$ ); Percutaneous coronary intervention without acute myocardial infarction (First wave: 6 vs 22 admissions per week, $\mathrm{p}<0.01$, reduction of $74 \%$; Second wave: 16 vs 23 admissions per week, $\mathrm{p}=0.01$, reduction of $31 \%$ ); Lower extremity arterial procedures (First wave: 2 vs 8 admissions per week, $\mathrm{p}<0.01$, reduction of $80 \%$; Second wave: 5 vs 8 admissions per week, $\mathrm{p}<0.01$, reduction of $42 \%$ ); Cardiac catheterization for other non-coronary conditions (First wave: 3 vs 16 admissions per week, $\mathrm{p}<0.01$, reduction of $80 \%$; Second wave: 8 vs 16 admissions per week, $\mathrm{p}<0.01$, reduction of $50 \%$ ); Other digestive system diagnoses (First wave: 3 vs 8 admissions per week, $p<0.01$, reduction of $68 \%$ ); Hip joint replacement (Second wave: 3 vs 9 admissions per week, $p<0.01$, reduction of $68 \%$ ); Knee joint replacement (First wave: 1 vs 9 admissions/week, $\mathrm{p}=0.02$, reduction of $88 \%$; Second wave: 3 vs 10 admissions per week, $\mathrm{p}<0.01$, reduction of $72 \%$ ); Uterine and adnexa procedures for non-malignancy except leiomyoma (First wave: 0 vs 8 admissions per week, $\mathrm{p}<0.01$, reduction of $100 \%$; Second wave: 2 vs 9 admissions per week, $\mathrm{p}<0.01$, reduction of 73\%); Alcohol and drug dependence with rehabilitation, detoxication, therapy (First wave: 0 vs 7 admissions per week, $\mathrm{p}<0.01$, reduction of $100 \%$; Second wave: 4 vs 8 admissions per week, $\mathrm{p}<0.01$, reduction of $42 \%$ ); Signs, symptoms and other factors influencing health status (Second wave: 5 vs 9 admissions per week, $\mathrm{p}<0.01$, reduction of 50\%); Other aftercare and convalescence (Second wave: 9 vs 18 admissions per week, $\mathrm{p}<0.01$, reduction of $48 \%$ ); Moderately extensive procedure unrelated to principal diagnosis (First wave: 2 vs 10 admissions per week, $p<0.01$, reduction of $79 \%$ ).

Average admissions per week did not statistically increase during the late-COVID period, compared to the peak-COVID period.

Some APRDRGs remained very stable across the different periods of the two waves of the pandemic: Appendectomy, Cesarean delivery, Vaginal delivery, Neonate birthweight $>2499$ g - normal newborn or neonate, Other chemotherapy.

\section{Analyses by Diagnosis (ICD I0-CM)}

Considering the thirty most frequent admission diagnoses according to ICD-10-CM, admissions did not statistically decrease during the early-COVID period (Table 3, 


\begin{tabular}{|c|c|c|c|c|c|c|c|c|c|c|c|c|c|c|c|c|c|c|c|}
\hline & & 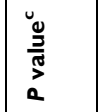 & ò & 今े & 今े & 。े & $\stackrel{\infty}{\circ}$ & oे & oे & oे & 吕 & 。 & $\stackrel{\infty}{0}$ & o̊. & oे & 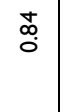 & oे & oे & ó \\
\hline & 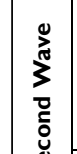 & فำ & 으 & n & $m$ & $\sigma$ & $\sigma$ & $m$ & $\sigma$ & $N$ & $\underline{\infty}$ & $\sigma$ & $\infty$ & 으 & $\circ$ & n & 0 & $\sigma$ & $N$ \\
\hline & 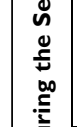 & 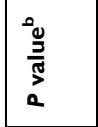 & o̊ & ત̃ & $\bar{o}$ & $\overline{\dot{Q}}$ & $\overline{\dot{Q}}$ & o̊̀ & $\frac{\infty}{0}$ & $\begin{array}{l}\overline{0} \\
\dot{\mathrm{v}}\end{array}$ & ơ & $\overline{\dot{O}}$ & 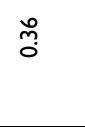 & $\overline{\stackrel{o}{v}}$ & $\frac{m}{0}$ & $\begin{array}{c}\text { \$ } \\
\text { D }\end{array}$ & for & $\overline{\grave{D}}$ & $\overline{\dot{D}}$ \\
\hline & 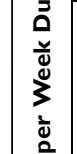 & 完 & 으 & t & $m$ & + & n & $m$ & + & $N$ & $\underline{0}$ & in & 0 & $\infty$ & in & 0 & 0 & $m$ & $m$ \\
\hline & 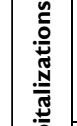 & 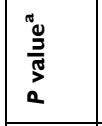 & $\stackrel{\infty}{\circ}$ & oे & 今̃ & 今̊ & 。े & สี & oे & $\stackrel{\infty}{\circ}$ & 今े & ঃ̊ & oे & $\stackrel{\infty}{\circ}$ & oे & 今े & ঙ્ণ & oे & 。े \\
\hline & 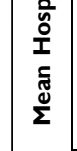 & | & 음 & r & 0 & $\infty$ & $\underline{\underline{n}}$ & + & $\sigma$ & $\circ$ & $\tilde{\lambda}$ & $n$ & $\infty$ & $=$ & r & 0 & in & $\infty$ & 으 \\
\hline & & 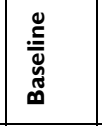 & $=$ & n & n & 으 & $=$ & $\wedge$ & $\infty$ & $\circ$ & $\ddot{\sim}$ & $\infty$ & $\infty$ & $\underline{\bullet}$ & $\infty$ & n & n & $\sigma$ & 으 \\
\hline & & 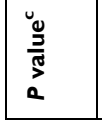 & $\begin{array}{l}\sigma \\
\sigma \\
\sigma\end{array}$ & ó & $\stackrel{\alpha}{\circ}$ & مे & 今े & oे & oे & ô & $\frac{\circ}{0}$ & م. & oे & ô & $\stackrel{\circ}{\circ}$ & ô & $\stackrel{\infty}{\circ}$ & $\stackrel{\infty}{\circ}$ & oे \\
\hline & 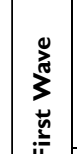 & نَ & $\infty$ & $m$ & $m$ & $N$ & + & $m$ & $N$ & $\sigma$ & \pm & $m$ & $N$ & n & 0 & $\sigma$ & + & $\sigma$ & $N$ \\
\hline & 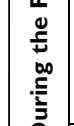 & 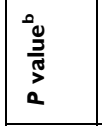 & $\stackrel{\circ}{\circ}$ & ō & $\bar{o}$ & $\overline{\stackrel{o}{v}}$ & $\overline{\stackrel{o}{\mathrm{v}}}$ & 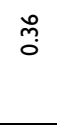 & $\overline{0}$ & $\overline{\bar{o}}$ & $\begin{array}{l}\overline{0} \\
\stackrel{\mathrm{v}}{ }\end{array}$ & $\begin{array}{l}\overline{0} \\
\dot{Q}\end{array}$ & $\bar{o}$ & $\overline{\stackrel{o}{\mathrm{v}}}$ & $\tilde{O}$ & $\stackrel{m}{0}$ & $\overline{\grave{D}}$ & $\stackrel{\text { to }}{0}$ & ठ̊. \\
\hline & 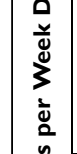 & نั & $\circ$ & - & $\sim$ & $N$ & $m$ & $\sigma$ & $m$ & $m$ & 0 & $\sim$ & - & $m$ & $\sigma$ & $m$ & $m$ & $N$ & - \\
\hline & 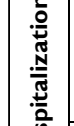 & 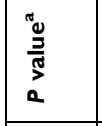 & $\stackrel{\infty}{\circ}$ & 苔 & $\stackrel{\infty}{\infty}$ & $\stackrel{\bar{\infty}}{\circ}$ & oे & 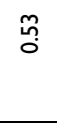 & oे & 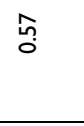 & ó & $\stackrel{\substack{\infty \\
0}}{0}$ & ò & à & $\stackrel{+}{\stackrel{t}{0}}$ & $\begin{array}{l}\infty \\
0 \\
0\end{array}$ & $\stackrel{\circ}{\circ}$ & oे & o. \\
\hline & 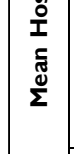 & 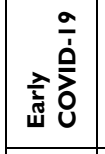 & $=$ & n & n & n & $=$ & $\simeq$ & $\infty$ & + & i & $\circ$ & $\infty$ & \pm & r & $N$ & $\circ$ & r & $\infty$ \\
\hline & & 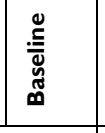 & $\simeq$ & n & $\infty$ & $\sigma$ & $\simeq$ & $\sigma$ & $\infty$ & $\circ$ & $\pi$ & $\infty$ & $\sigma$ & $\underline{0}$ & $\sigma$ & in & $\infty$ & $\infty$ & $\sigma$ \\
\hline & 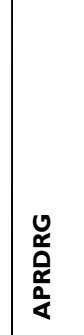 & & 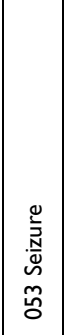 & 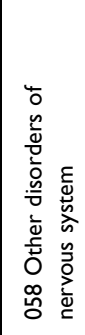 & 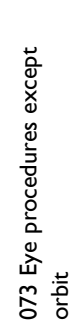 & 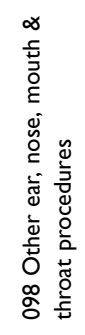 & 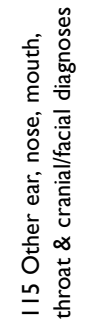 & 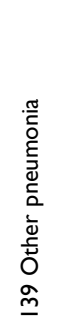 & 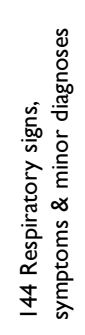 & 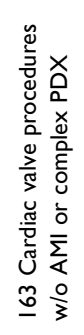 & 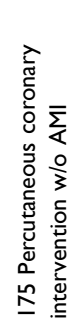 & 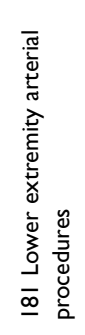 & 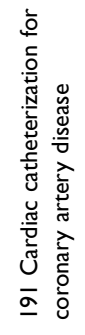 & 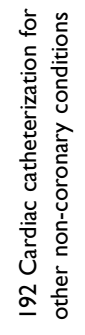 & 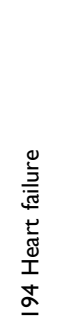 & 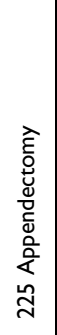 & 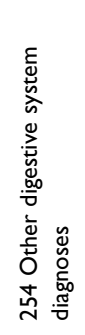 & 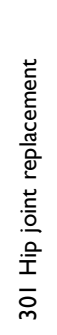 & 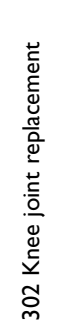 \\
\hline
\end{tabular}




\begin{tabular}{|c|c|c|c|c|c|c|c|c|c|c|c|c|c|c|}
\hline \multirow{7}{*}{ 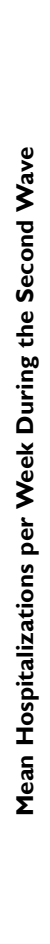 } & 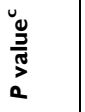 & 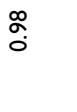 & مٌ & o̊ & 哭 & $\underset{\infty}{\widetilde{\infty}}$ & o̊ & ì & o̊ & S. & & ஃ̊ & 䒘 & $\stackrel{2}{\circ}$ \\
\hline & 选 & $m$ & in & $\sigma$ & $\sigma$ & 0 & $\bar{N}$ & \pm & $\underline{0}$ & $m$ & 0 & 0 & 0 & $\sigma$ \\
\hline & 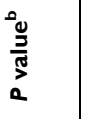 & ڤึ & §̊. & ठ̊. & $\begin{array}{l}\overline{\dot{O}} \\
\dot{\mathrm{v}}\end{array}$ & o̊ & $\stackrel{\infty}{\infty}$ & o. & o. & ণ্ণ & $\begin{array}{l}\bar{\partial} \\
\dot{v}\end{array}$ & 产 & $\begin{array}{l}\overline{\dot{\theta}} \\
\dot{v}\end{array}$ & י্ণ \\
\hline & 产 & $\sigma$ & $\sigma$ & $\sigma$ & $N$ & $\infty$ & $\tilde{\sim}$ & $\stackrel{\infty}{\sim}$ & $\simeq$ & $\sigma$ & $\sigma$ & in & $\sigma$ & N \\
\hline & 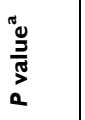 & $\stackrel{n}{0}$ & 。. & 令 & $\stackrel{\alpha}{\alpha}$ & 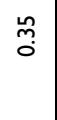 & $\hat{o}$ & $\stackrel{\infty}{\circ}$ & مٌ & ‡. & م. & 。. & ô & o̊ \\
\hline & 窝 & $\infty$ & $\infty$ & in & $\infty$ & $\circ$ & $\approx$ & $\stackrel{2}{\sim}$ & $\underline{\infty}$ & in & $\wedge$ & $\sigma$ & $\underline{\infty}$ & $\sigma$ \\
\hline & 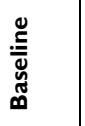 & 0 & $\infty$ & $\wedge$ & $\sigma$ & $\infty$ & $\stackrel{\sim}{\sim}$ & নे & 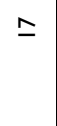 & r & $\infty$ & $\sigma$ & $\underline{\infty}$ & 으 \\
\hline \multirow{7}{*}{ 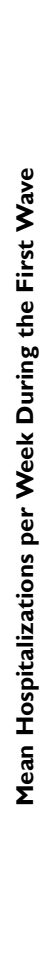 } & 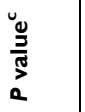 & ֻ̊̊. & o. & o. & & o̊ & ô. & ১. & مे & ô. & & ô & iñ & $\stackrel{n}{\circ}$ \\
\hline & فे & $\sigma$ & $\sigma$ & $\sigma$ & $m$ & $n$ & $\stackrel{\sim}{\sim}$ & సे & \pm & $\sigma$ & 0 & $\sigma$ & $\simeq$ & in \\
\hline & 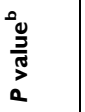 & $\overline{0}$ & 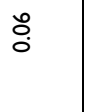 & 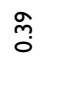 & $\stackrel{n}{0}$ & o̊ & 今े & og & ơ & बू. & $\overline{\grave{O}}$ & $\stackrel{\circ}{0}$ & $\stackrel{\circ}{\circ}$ & $\overline{\grave{D}}$ \\
\hline & 商 & - & $m$ & $m$ & 0 & N & $\approx$ & $\stackrel{2}{\pi}$ & $\underline{0}$ & in & 0 & $\sim$ & N & $\sim$ \\
\hline & 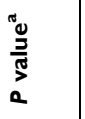 & ó & $i_{0}^{+}$ & ì & 尽 & $\stackrel{\widehat{\infty}}{\circ}$ & $\stackrel{\circ}{\circ}$ & مُ & م. & $\stackrel{\infty}{\circ}$ & oे & ô. & ô. & $\frac{\infty}{0}$ \\
\hline & 﨎 & $\wedge$ & in & $m$ & $\circ$ & 으 & $\underline{a}$ & $\hat{\lambda}$ & $\underline{\bullet}$ & in & $\infty$ & $\infty$ & $\underline{m}$ & $\wedge$ \\
\hline & 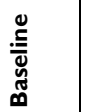 & $\wedge$ & $\infty$ & in & $\infty$ & $\infty$ & $\pi$ & $\stackrel{\sim}{\pi}$ & \pm & $\circ$ & n & $=$ & $\underline{\text { 음 }}$ & 으 \\
\hline $\begin{array}{l}0 \\
\frac{0}{0} \\
\frac{\alpha}{\alpha} \\
\frac{\alpha}{\alpha}\end{array}$ & & 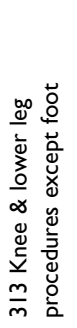 & 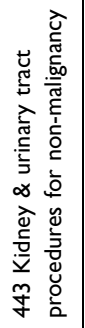 & 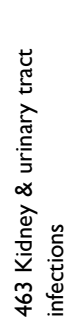 & 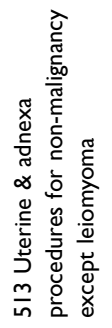 & 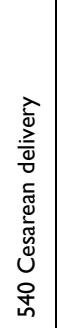 & 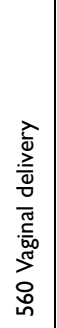 & 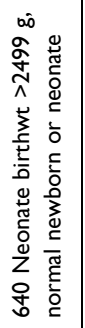 & 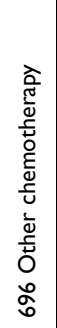 & 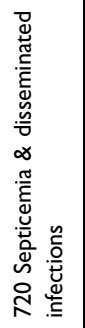 & 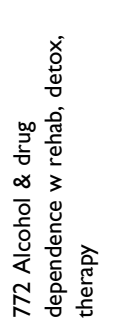 & 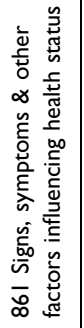 & 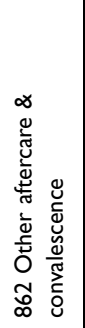 & 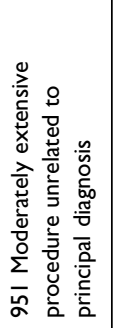 \\
\hline
\end{tabular}




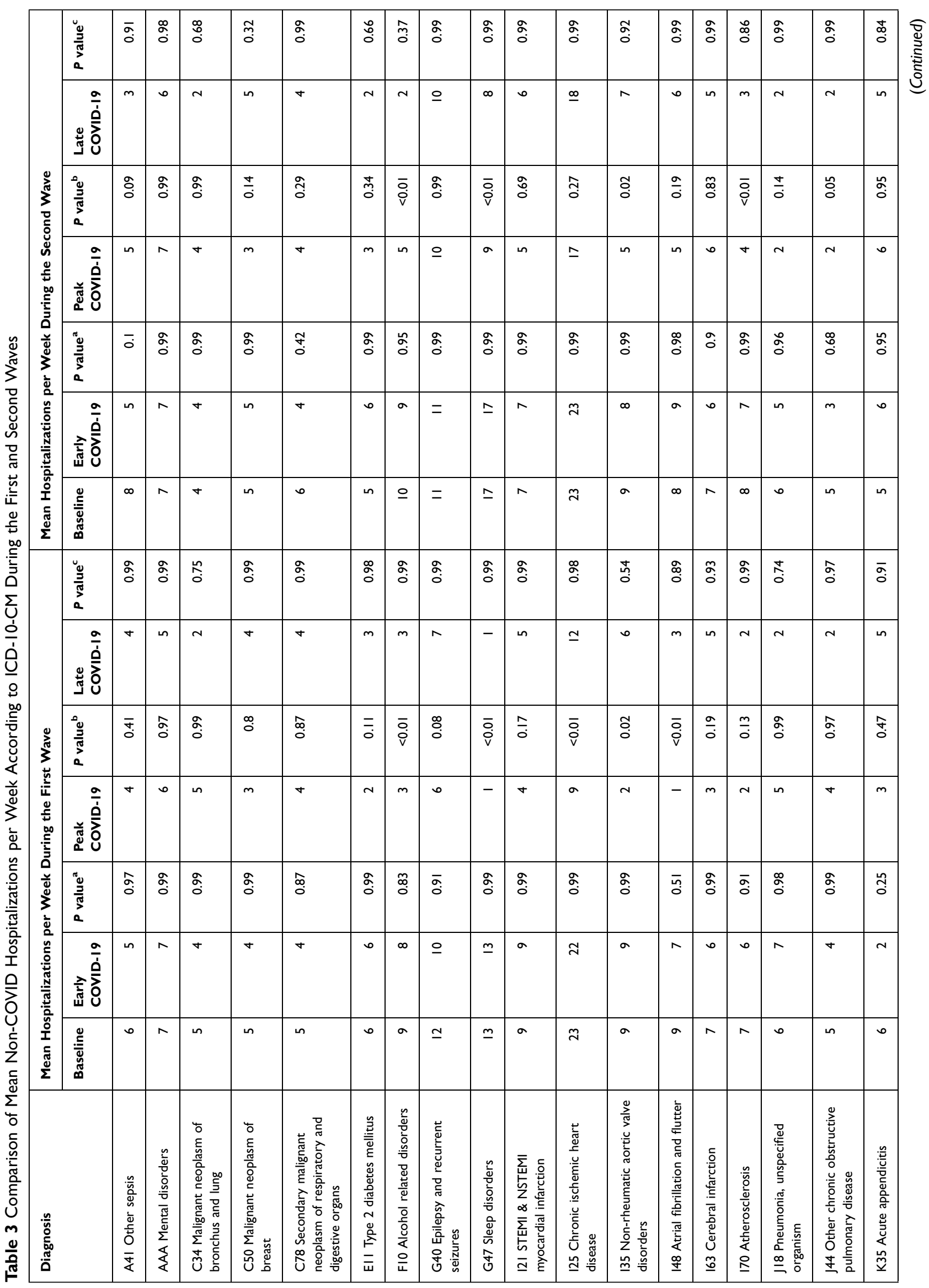




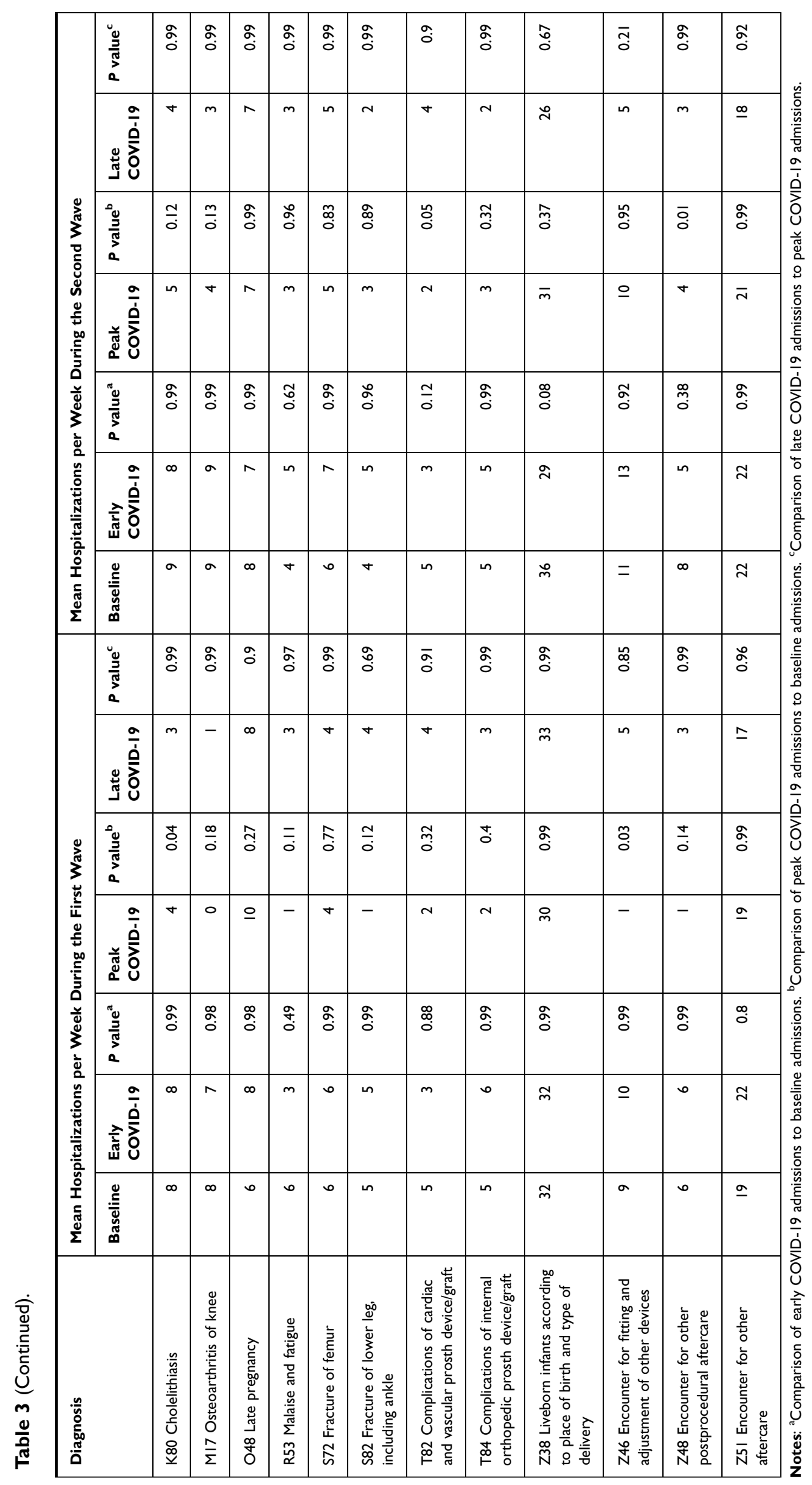


Figure 1C and D [Supplementary File]). The diseases whose average admissions per week statistically decreased during the peak-COVID period compared to the baseline preCOVID period were: Alcohol related disorders (First wave: 3 vs 9 admissions per week, $\mathrm{p}<0.01$, reduction of $73 \%$; Second wave: 5 vs 10 admissions per week, $\mathrm{p}<0.01$, reduction of 57\%); Sleep disorders (First wave: 1 vs 13 admissions per week, $\mathrm{p}<0.01$, reduction of 92\%; Second wave: 9 vs 17 admissions per week, $\mathrm{p}<0.01$, reduction of $49 \%$ ); Chronic ischemic heart disease (First wave: 9 vs 23 admissions per week, $\mathrm{p}<0.01$, reduction of $61 \%$ ); Atrial fibrillation and flutter (First wave: 1 vs 9 admissions per week, $\mathrm{p}<0.01$, reduction of $86 \%$ ); Atherosclerosis (Second wave: 4 vs 8 admissions per week, $\mathrm{p}<0.01$, reduction of $47 \%$ ).

There was no significant increase in admissions by diagnosis during the late-COVID period, compared to the peak-COVID period.

Some admissions rates remained particularly stable across the different COVID-period: admissions for STelevation of myocardial infarction (STEMI) and nonSTEMI, cerebral infarction, malignant neoplasm (bronchus and lung, breast, and secondary neoplasm for respiratory and digestive organs), mental diseases, hip fractures, liveborn infants.

\section{Discussion}

The first main findings of our study was that non-COVID admissions (all categories) were reduced during the COVID crisis by around 39\% during the first wave and $29 \%$ during the second wave compared to 2018 and 2019.

Our results on non-COVID in-hospital admissions are in line with those published in the literature. ${ }^{9-16}$ Birkmeyer et al found in a large study of approximately one million medical admissions in the US that declines in non-COVID admissions from the first two months of COVID crisis exceeded $20 \%$ for all primary admission diagnoses. ${ }^{9}$ Kapsner et al found that overall in-patient hospital admissions in Germany decreased by $35 \%$ in the first month and by $30.3 \%$ in the second month after the lockdown announcement during the first wave compared to $2018 .{ }^{10}$ Caminiti et al found in Italy that approximately one third of the overall admissions for non-COVID in 2020 vs 2019 was reduced. $^{11}$ Blecker et al found a reduction of non-COVID admissions by $32 \%$ and $43 \%$ during de COVID first wave compared to 2018 and 2019 respectively. ${ }^{17}$ Kuhlen et al reported also a decrease of 42.7\% in-hospital admissions between March 3 and April 19, 2020 in comparison with $2019 .{ }^{15}$ Recently,
Bodilsen et al in a Danish nationwide cohort study involving more than 1 million of hospital admissions, found that compared with the pre-COVID baseline period (mean hospital admission rate 204.1 per 100,000 /week), the overall hospital admission rate for non COVID-19 conditions was reduced to 142.8 per $100,000 /$ week after the first national lockdown, followed by a gradual return to baseline state until the second lockdown when it decreased to 158.3 per $100,000 /$ week. $^{16}$

The second most interesting findings of our study is that the decrease of admissions (non-COVID) was statistically significant during the peak-COVID and late-COVID periods but not the early-COVID period during the two waves compare to pre-COVID period. The decrease was in most of the type of admissions but some were significantly affected by the crisis depending on restrictive measures undertaken at the country and hospital levels. Blecker et $\mathrm{al}^{17}$ found also similar results with no difference in rate of hospitalization between the early COVID-19 period and the baseline period (604.3 vs 584.5 per week; $P=0.19$ ), and a decreased during the peak COVID-19 period (247.0 per week; $P<0.001$ ). They found also that the rate of hospitalization decreased across all categories of diagnoses during the peak COVID-19 period. $^{17}$

Very interestingly, our analyses found that myocardial infarction (STEMI, NSTEMI), cerebral infarction and cancer were not statistically reduced during the two waves of COVID pandemic compared to the pre-COVID period in our hospital. Our results contrasted with those published in the literature ${ }^{10,14,15,17-21}$ and were somewhat similar to a recent study by Solomon et al. ${ }^{22}$ Kapsner et al ${ }^{10}$ found that there were a reduction of $38.7 \%$ of admissions for myocardial infarction (736 to 451) from 2018 to 2020 and $19.6 \%$ of admissions for stroke (1260 to 1013$).{ }^{10}$ Günster et $\mathrm{al}^{14}$ found a decrease in 2020 admissions for myocardial infarction compared to 2019. Rodríguez-Leor et al reported in Spain for STEMI-related procedures across 73 sites in Spain a reduction of $40 \% .^{13}$ Recently, Mafham et al, in a large National Health Services (NHS) database in England, found a reduction in coronary syndrome admissions in March 2020 of 40\% compared to 2019 , but this reduction had slowed down in the following months and was not more than $16 \%$ at the end of May. It should be noted that this reduction concerned all types of acute coronary syndromes, including STEMI and NSTEMI but the reduction was much greater for NSTEMI. There were $21 \%$ and $37 \%$ reduction of the number of percutaneous coronary intervention (PCI) 
procedures for STEMI and non STEMI respectively at the end of March. ${ }^{20}$ Similarly, a French study of 1167 patients admitted for acute myocardial infarction within 48 hours with an equivalent number of STEMI and NSTEMI, found a $30 \%$ reduction in acute myocardial infarction admissions between the post-lockdown period and pre-lockdown. More precisely there was a reduction of $24 \%$ and $35 \%$ for STEMI and NSTEMI respectively. The reduction was observed with similar trends according to sex, risk factors and regional prevalence of hospital admissions for COVID-19. ${ }^{21}$

Concerning cancer care, many other groups reported a reduction of in-hospital admissions and surgeries between $8-39 \%$ for lung cancer ${ }^{10,15,22}$ and between 2$14 \%$ for brain cancer. ${ }^{10,15}$

The difference seen between our results and those published in the literature in terms of the reduction of these critical diseases can be explain partially by our hospital policy (coronary unit, cardiac intensive care and cardiac surgery unit, stroke unit, oncological one-day clinic and ward were fully open) and our careful selection of emergency procedures (each physician was asked to select himself on case by case and on week basis admissions requiring urgent care). Indeed, we found a significant decreased in cardiac valve procedures without acute myocardial infarction or complex procedures and diagnoses (Second wave: 2 vs 6 admissions per week, $\mathrm{p}<0.01$, reduction of $69 \%$ ); Percutaneous coronary intervention without acute myocardial infarction (First wave: 6 vs 22 admissions per week, $\mathrm{p}<0.01$, reduction of $74 \%$; Second wave: 16 vs 23 admissions per week, $\mathrm{p}=0.02$, reduction of $31 \%$ ); Cardiac catheterization for other non-coronary conditions (First wave: 3 vs 16 admissions per week, $\mathrm{p}<0.01$, reduction of $80 \%$; Second wave: 8 vs 16 admissions per week, $\mathrm{p}<0.01$, reduction of $50 \%$ ). More recently, data from the Kaiser Permanente Northern California shows that the reduction observed at the beginning of the pandemic in March for acute myocardial infarctions and strokes was not observed during the second wave of the pandemic. A modest decline was observed for stroke alerts during the summer COVID-19 surge but quickly rebounded. ${ }^{22}$

Our carefully selection of emergency was also illustrated by the fact that surgeries for hip fractures were not reduced during the two waves, in contrast to surgeries of knee and hip replacement. Indeed, we found a statistically significant decrease in average number of admissions per week of hip joint replacement (Second wave: 3 vs 9 admissions per week, $\mathrm{p}<0.01$, reduction of
68\%); knee joint replacement (First wave: 1 vs 9 admissions/week, $\mathrm{p}=0.02$, reduction of $88 \%$; Second wave: 3 vs 10 admissions per week, $\mathrm{p}<0.01$, reduction of $72 \%$ ) during waves. Our results of hip fracture surgeries and hip and knee replacement surgeries are similar to those found in Germany by Kapsner et $\mathrm{al}^{10}$ and Günster et al. ${ }^{14}$ Kapsner et $\mathrm{al}^{10}$ in Germany found that in the first month of the lockdown there was no reduction in the number of admissions for hip fractures while there was an $82 \%$ reduction for primary hip and knee prostheses. ${ }^{10}$ This was not always the case in some other European countries, ${ }^{23-25}$ confirming the effect of different hospitals and countries policies on the impact of COVID pandemic on nonCOVID in-hospital admissions.

This study has limitations. First, it is a retrospective analysis based on observational data collected in a single center despite the fact that it is the largest teaching hospital in Brussels. Secondly, our data represented a partial picture of all Belgian academic centers or general hospitals. Thirdly, despite using different type of classification (APRDRG, ICD10-M) we cannot exclude bias and limitations inherent to those classifications. Finally, our study is not designed to analyse the impact of the reduction of nonCOVID admissions on the quality of life or the outcomes of those patients. Nevertheless, our study provided an excellent overview of the impact of COVID pandemic on non COVID-in-hospital admissions and this impact depends on lockdown decision at the country level and also depends on the local policy at the hospital level.

\section{Conclusions}

Our study shows that non-COVID in-hospital admissions rates were substantially reduced following the national COVID-19 lockdown in Belgium during the COVID pandemic specially during the peak and late COVID period of the first and second waves. The reduction concernsw all non-COVID category of admissions, however in our hospital, critical clinical situations such as cancer, myocardial and cerebral infarctions were not statistically reduced compared to what was described in the literature. This is very interesting because deferring those critical situations is expected to severely impair quality of life and clinical outcomes. It would be interesting to see whether this was only the situation in our hospital or the global situation at the national level.

\section{Disclosure}

The authors report no conflicts of interest in this work. 


\section{References}

1. Kansagra AP, Goyal MS, Hamilton S, Albers GW. Collateral effect of Covid-19 on stroke evaluation in the United States. $N$ Engl J Med. 2020;23(383):400-401. doi:10.1056/NEJMc2014816

2. Die Bundesregierung. Sozialkontakte vermeiden, Ausbreitung verlangsamen; 2020. Available from: https://www.bundesregierung. de/breg-de/themen/coronavirus/mpk-1730186. Accessed August 7, 2020.

3. Hartnett KP, Kite-Powell A, DeVies J, et al. Impact of the COVID-19 pandemic on emergency department visits - United States, January 1, 2019-May 30, 2020. MMWR Morb Mortal Wkly Rep. 2020;69 (23):699-704. doi:10.15585/mmwr.mm6923e1

4. COVIDSurg Collaborative. Elective surgery cancellations due to the COVID-19 pandemic: global predictive modelling to inform surgical recovery plans: elective surgery during the SARS-CoV-2 pandemic. Br J Surg. 2020;107:1440-1449.

5. Baum A, Schwartz MD. Admissions to veterans affairs hospitals for emergency conditions during the COVID-19 pandemic. JAMA. 2020;324(1):96-99. doi:10.1001/jama.2020.9972

6. Siegler JE, Heslin ME, Thau L, Smith A, Jovin TG. Falling stroke rates during COVID-19 pandemic at a comprehensive stroke center J Stroke Cerebrovasc Dis. 2020;29(8):104953. doi:10.1016/j. jstrokecerebrovasdis.2020.104953

7. Solomon MD, McNulty EJ, Rana JS, et al. The COVID-19 pandemic and the incidence of acute myocardial infarction. $N$ Engl $J$ Med 2020;383(7):691-693. doi:10.1056/NEJMc2015630

8. Chernew ME, Fendrick AM, Armbrester K, de Brantes F. COVID-19 effects on care volumes: what they might mean and how we might respond. Health Aff Blog. 2020. Available from: https://www.healthaffairs.org/do/10.1377/hblog20200702.788062/ full/. Accessed October 21, 2021.

9. Birkmeyer JD, Barnato A, Birkmeyer N, Bessler R, Skinner J. The impact of the COVID-19 pandemic on hospital admissions in the United States. Health Aff. 2020;39(11):2010-2017. doi:10.1377/ hlthaff.2020.00980

10. Kapsner LA, Kampf MO, Seuchter SA, et al. Reduced rate of inpatient hospital admissions in 18 German University Hospitals during the COVID-19 lockdown. Front Public Health. 2021;8:594117. doi:10.3389/fpubh.2020.594117

11. Caminiti C, Maglietta G, Meschi T, Ticinesi A, Silva M, Sverzellati N. Effects of the COVID-19 epidemic on hospital admissions for non-communicable diseases in a Large Italian University-Hospital: a Descriptive Case-Series Study. J Clin Med. 2021;10(4):880. doi:10.3390/jcm10040880

12. Hoyer C, Ebert A, Huttner HB, et al. Acute stroke in times of the covid-19 pandemic: a multicenter study. Stroke. 2020;51:2224-2227. doi:10.1161/STROKEAHA.120.030395

13. Rodríguez-Leor $\mathrm{O}$, Cid-ãlvarez $\mathrm{B}$, Ojeda $\mathrm{S}$, et al. Impact of the COVID-19 pandemic on interventional cardiology activity in Spain. REC Interv Cardiol. 2020;2:4060.
14. Günster C, Drogan D, Hentschker C, et al. WIdO-Report: Entwicklung der Krankenhausfallzahlen während des Coronavirus-Lockdowns: Nach ICD-10-Diagnosekapiteln und ausgewähltenBehandlungsanlässen. Wissenschaftliches Institut Der AOK (WldO); 2020.

15. Kuhlen R, Schmithausen D, Winklmair C, Schick J, Scriba P. The effects of the COVID-19 pandemic and lockdown on routine hospital care for other illnesses. Dtsch Arztebl Int. 2020;117:488-489.

16. Bodilsen J, Nielsen PB, Søgaard M, et al. Hospital admission and mortality rates for non-covid diseases in Denmark during covid-19 pandemic: nationwide population based cohort study. BMJ. 2021;373:n1135. doi:10.1136/bmj.n1135

17. Blecker S, Jones SA, Petrilli CM, et al. Hospitalizations for chronic disease and acute conditions in the time of COVID-19. JAMA Intern Med. 2021;181(2):269-271. PMID: 33104158; PMCID: PMC7589070. doi:10.1001/jamainternmed.2020.3978

18. Garcia S, Albaghdadi MS, Meraj PM, et al. Reduction in ST-segment elevation cardiac catheterization laboratory activations in the United States during COVID-19 pandemic. $J$ Am Coll Cardiol. 2020;75:2871-2872. doi:10.1016/j.jacc.2020.04.011

19. Bodanowitz J. Herzinfarkt: deutlich weniger Krankenhauseinweisungen im März Sonderanalyse der DAK-Gesundheit belegt Rückgang der stationären Aufnahmen um 25 Prozent; 2020. Available from: https:// www.dak.de/dak/bundesthemen/herzinfarkt-2259192.html\#/. Accessed August 8, 2020.

20. Mafham MM, Spata E, Goldacre R, et al. COVID-19 pandemic and admission rates for and management of acute coronary syndromes in England. Lancet. 2020;396(10248):381-389. doi:10.1016/S01406736(20)31356-8

21. Mesnier J, Cottin Y, Coste P, et al. Hospital admissions for acute myocardial infarction before and after lockdown according to regional prevalence of COVID-19 and patient profile in France: a registry study. Lancet Public Health. 2020;5(10):e536-e542. doi:10.1016/ S2468-2667(20)30188-2

22. Solomon MD, Nguyen-Huynh M, Leong TK, et al. Changes in patterns of hospital visits for acute myocardial infarction or ischemic stroke during COVID-19 surges. JAMA. 2021;2:e218414.

23. Rattka M, Baumhardt M, Dreyhaupt J, et al. 31 days of COVID-19cardiac events during restriction of public life - a comparative study. Clin Res Cardiol. 2020;109:1476-1482. doi:10.1007/s00392-02001681-2

24. Hernigou J, Morel X, Callewier A, Bath O, Hernigou P. Staying home during "COVID-19" decreased fractures, but trauma did not quarantine in one hundred and twelve adults and twenty eight children and the "tsunami of recommendations" could not lockdown twelve elective operations. Int Orthop. 2020;44:1473-1480. doi:10.1007/s00264-020-04619-5

25. Maniscalco P, Poggiali E, Quattrini F, et al. Proximal femur fractures in COVID-19 emergency: the experience of two orthopedics and traumatology departments in the first eight weeks of the Italian epidemic. Acta Biomed. 2020;91:89-96.
International Journal of General Medicine

\section{Publish your work in this journal}

The International Journal of General Medicine is an international, peer-reviewed open-access journal that focuses on general and internal medicine, pathogenesis, epidemiology, diagnosis, monitoring and treatment protocols. The journal is characterized by the rapid reporting of reviews, original research and clinical studies across all disease areas. The manuscript management system is completely online and includes a very quick and fair peer-review system, which is all easy to use. Visit http://www.dovepress.com/ testimonials.php to read real quotes from published authors. 